\title{
Massive upper gastrointestinal bleeding and diffuse benign gastric inflammatory hyperplastic polyps in a 19 year old patient: a case report
}

\author{
HYASINTA M. JAKA ${ }^{*}$, MARIAM MIRAMBO ${ }^{2}$, PETER RAMBAU ${ }^{3}$ and PHILLIPO L. CHALYA ${ }^{2}$ \\ ${ }^{1}$ Department of Internal medicine, Catholic University of Health and Allied Sciences-Bugando, Mwanza, Tanzania \\ ${ }^{3}$ Department of Microbiology and Immunology, Catholic University of Health and Allied Sciences-Bugando, \\ Mwanza, Tanzania \\ \$Department of Pathology, Catholic University of Health and Allied Sciences-Bugando, Mwanza, Tanzania \\ ${ }^{2}$ Department of Surgery, Catholic University of Health and Allied Sciences-Bugando, Mwanza, Tanzania
}

\begin{abstract}
Benign gastric inflammatory hyperplasic polyps are benign lesions that rarely occur in young age. We report a case of diffuse benign gastric inflammatory hyperplastic polyps in a 19 year old male patient who presented with cough, nausea, and haematemesis. In the presented case symptoms such as nausea and vomiting are non specific and may complicate in reaching a prompt diagnosis. We also highlight the importance of gastrointestinal tract (GIT) endoscopy in the diagnosis of GIT diseases.
\end{abstract}

Keywords: hyperplastic polyps, endoscopy, gastrointestinal tract, bleeding, Tanzania

\section{Introduction}

Diffuse benign inflammatory hyperplasic polyps also known as diffuse gastric polyposis is a rare form of benign lesion with only a few cases being reported (Hu et al., 2002). It may occur throughout the gastrointestinal tract (GIT), commonly in aged people with average of 60 years (Chongsrisawat et al., 2004). It may also be part of polyposis syndromes associated with genetic factors (Jayawardena et al., 2008) especially in young aged patients. There is little information about the occurrence of gastric inflammatory polyps in young aged patients in our settings. Here, we report a case of diffuse benign inflammatory hyperplasic polyps in a young adult patient who was successfully diagnosed and managed through GIT endoscopy and surgical resection respectively.

\section{Case presentation}

A 19 year old young male of African descent presented to Bugando Medical Centre with a fourmonth history of productive cough and on and off haematemesis. He also reported to have black stool (melaena) for more than 4 months associated with episodes of syncope twice. The cough was worse during the day time and had no associated or precipitating factors. There was no past history of schistosomiasis, jaundice or bleeding tendencies. However, he had epilepsy since childhood and had a positive family history of epilepsy on his paternal side. Last convulsive episode was the day of admission. On admission he was found to be alert, weak, not dyspnoeic pale and afebrile $\left(36^{\circ} \mathrm{C}\right)$. Blood pressure was $88 / 47 \mathrm{mmHg}$, pulse rate was $108 \mathrm{bpm}$ and respiratory system was remarkable with no any significant findings. He had tonic clonic seizures with left sided body weakness associated with asymmetrical pupil reactions to light. Abdominal examination had tender epigastric region with no palpable mass. Rectal examination revealed normal rectal mucosa with no rectal mass and the gloves stained with black tally stool.

Laboratory investigation revealed negative results for malaria parasites and two times smear negative for acid fast bacilli (AFB). Sputum culture revealed normal flora of respiratory tract. The abdominal ultrasound revealed nothing significant and the chest X-ray was normal. Biochemical analysis showed all parameters were essentially normal. Haematological examination revealed haemoglobin $(\mathrm{Hb})$ of $8.6 \mathrm{~g} / \mathrm{dl}$ (anaemic), packed cell volume (PCV) 31.1\%, red blood cell (RBC) count $4.9 \times 10^{12} / \mathrm{L}$, erythrocyte sedimentation rate (ESR) $102 \mathrm{~mm} / \mathrm{hr}$, mean corpuscular

\footnotetext{
${ }^{*}$ Correspondence: Dr. Hyasinta M. Jaka: hjaka2@hotmail.com
} 
haemoglobin (MCH) 17.7pG, mean corpuscular volume (MCV) 63.9fL, mean corpuscular haemoglobin concentration (MCHC) $27.7 \%$ and total white blood cell (WBC) count was $6.22 \times 10^{9} / \mathrm{L}$. Other cells such as neutrophils $73 \%$, eosinophils $3.62 \%$, basophils $1.29 \%$, lymphocytes $17.1 \%$ and monocytes $6.18 \%$. Almost all haematological parameters examined showed there was microcytic anaemia. The Prothrombin time was within the normal ranges.

The emergency oesophago-gastroduodenoscopy was done and revealed presence of cauliflower friable lesions extending from the antrum to the middle portion of the corpus. Lesions were numerous diffuse polyps of varying size and shape. Histopathological examination of endoscopic biopsy confirmed the presence of benign gastric inflammatory hyperplastic polyps. We were unable to isolate Helicobacter pylori in biopsy specimens. The colonoscopy was done and revealed normal findings.

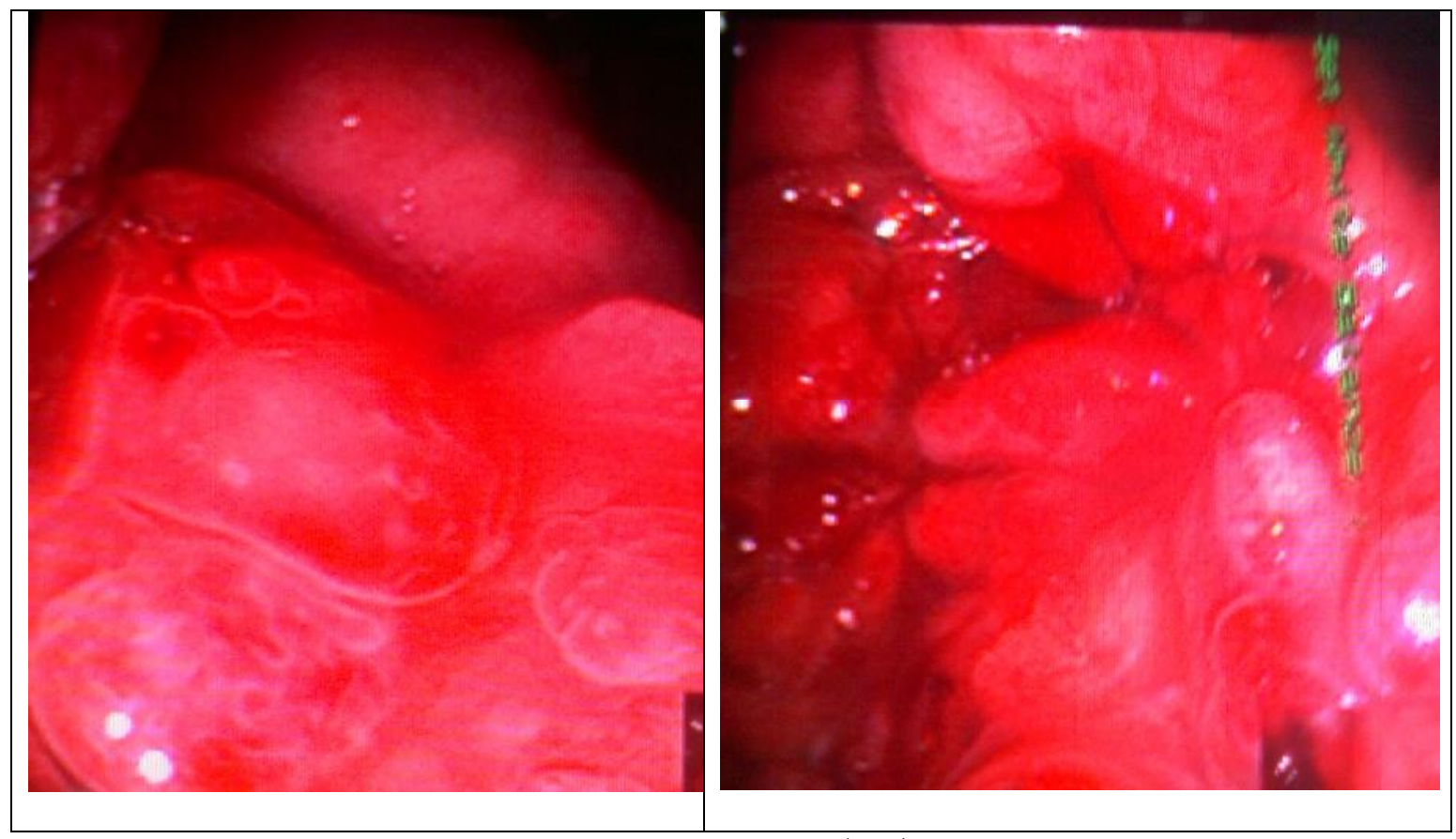

Figure1. Large lobulated polyps at gastric region (left) and numerous diffuse polyps of various size in the gastric region

On admission, he started with intravenous normal saline and he received two units of blood transfusion. The patients also received injection Diazepam 10mg and phenobabitone $25 \mathrm{mg}$ was given orally to control seizures and aggressiveness attacks. The patient became stable and he was transferred to surgical ward where the laparatomy was done. During laparatomy a cauliflower friable lesion extending from the antrum to the middle portion of the corpus was observed and resected (Figure 1 and 2). After 10 days of admission, the patient recovered successfully and continued to attend the clinic for check-ups. The consent to publish this case report was obtained from the patient.

\section{Discussion}

Gastric polyps are uncommon occurrence (Scott-Conner., 2006) with incidence of less than $1 \%$. (Jayawardena et al., 2008). Some cases have been reported in Europe and Asia with variation in prevalence in different countries (Carmack et al., 2009). Aetiology is not well established but it has been associated with inflamed and atropic gastric mucosa (Dirschmid, et al., 2006). Lesions often originate from the submucosal tissue and progress into a polypoid mass (Maves et al., 1989; Gonul 
et al., 2004; Chongsrisawat et al., 2004). In our patient polyps occurred more in the antrum than in other parts of the stomach. They are often multiple and usually smooth, dome-shaped with small diameters. In some cases they may reach larger dimensions and become lobulated and pedunculated (Abraham et al., 2001). Patients with this type of lesions often present late with abdominal pain, palpable abdominal mass, haematemesis, anorexia, nausea and gastrointestinal bleeding. However some patients may present with only few of these features at advanced stage of the disease and in young aged patients symptoms are non specific (Chongsrisawat et al., 2004, Badriul et al., 2007).In our case, the tumour was not palpable and the patient was anaemic. Similar observation was seen case reported by Jayawardena et al in 2008. Some features observed in other cases are not similar to what we observed in our patient. This suggest that gastric inflammatory hyperplastic polyps may present with various signs and endoscopic examination remain important tool for identification of gastric tumours as it provides a minimally invasive approach to diagnosis and treatment (Jayawardena et al., 2008)

Since these tumours in young age are relatively rare, is not commonly suspected or considered in the differential diagnosis of the Upper GIT bleeding. Diagnosis is often difficult since the initial symptoms may be non-specific; therefore high index of suspicion is needed. Familial history of cancer can be considered and may be useful during diagnosis especially when this type of lesion is suspected in young aged patients. It is therefore important to take a detailed family history during diagnosis of gastric diseases especially in young aged patients. However, we were unable to link genetic abnormalities and development of gastric inflammatory hyperplastic lesion in this patient due to limited resources. In our settings genetic investigations is not often done due to limited resources and financial limitations in most of our patients.

Endoscopic examination is affordable to some patients and is considered in unusual and complicated cases that need prompt diagnosis. On the other side abdominal ultrasound has been useful in diagnosing GIT diseases but sometimes it may give false negative results. In our case abdominal ultrasound revealed no significant findings but most of blood parameters such as HB, $\mathrm{PCV}, \mathrm{MCV}, \mathrm{MCH}$, and $\mathrm{MCHC}$ were below the normal ranges with exception of ESR due to anaemia he has. The haematological abnormalities observed in this patients prompted investigation of the cause of $\mathrm{Gl}$ bleeding which enables realization of the diffuse benign lesion. In this context care should be taken and all possible approaches should be considered during diagnosis of upper GIT bleeding especially in young aged patients.

In conclusion, upper GIT endoscopy is crucial in diagnosing GIT diseases especially in young aged patients. As emphasized in this case, diffuse gastric inflammatory hyperplastic polyps can also occur in young patients and it is therefore important to consider possibility of these lesions when diagnosing GIT diseases especially the upper GIT Bleeding in this age group.

\section{Ethical consideration}

Written informed consent was obtained from the patient's relatives for publication of this case report and accompanying images.

\section{References}

Abraham, S.C., Singh, V.K. \& Yardley, J.H. (2001) Hyperplastic polyps of the stomach: associations with histologic patterns of gastritis and gastric atrophy. American Journal of Surgical Pathology 25, 500-507.

Badriul, H. \& Tatang, P. (2007) Gastric Inflammatory fibroid polyp in children. The Indonesian Journal of Gastroenterology, Hepatology, and Digestive Endoscopy 8, 63-66.

Carmack, S.W., Genta, R.M., Graham, D.Y. \& Lauwers, G.Y. (2009) Management of gastric polyps: a pathology based guide for gastroenterologists. Nature Reviews Gastroenterology \& Hepatology 6, 331-341. 
Chongsrisawat, V., Yimyeam, P., Wisedopas, N., Viravaidya, D. \& Poovorawan, Y. (2004) Unusual manifestations of gastric inflammatory fibroid polyp in a child. World Journal of Gastroenterology 10, 460-462.

Dirschmid, K., Platz-Baudin, C. \& Stolte, M. (2006) Why is the hyperplastic polyp a marker for the precancerous condition of the gastric mucosa? Virchows Archives 448, 80-84.

Gonul, I.I., Erdem, O. \& Ataoglu, O. (2004) Inflammatory fibroid polyp of the ileum causing intussusception: a case report. Turkey Journal of Gastroenterology 15, 49-62.

Hu, T.L., Hsu, J.T., Chen, H.M. \& Chen, M.F. (2002) Diffuse gastric polyposis: report of a case. Journal of Formosa Medical Association 101, 712-714.

Jayawardena, S., Anandacoomaraswamy, D., Burzyantseva, O. \& Abdullah, M. (2008) Case Report: Isolated diffuse hyperplastic gastric polyposis presenting with severe anemia. Cases Journal $1,1-3$.

Maves, K.M., Johnson, I.F., Bove, K., Mallot, R.L. (1989) Gastric inflammatory pseudotumor in children. Radiology 173, 381-383.

Scott-Conner, C.EH. (2006) Benign Gastric Tumors. eMedicine 